\title{
Neutrophil-to-lymphocyte ratio is a marker for acute kidney injury progression and mortality in critically ill populations: a population-based, multi-institutional study
}

\author{
Jia-Jin Chen ${ }^{1} \cdot$ George Kuo $^{1} \cdot$ Pei-Chun Fan ${ }^{1,2} \cdot$ Tao-Han Lee $^{1} \cdot$ Chieh-Li Yen $^{1} \cdot$ Cheng-Chia Lee $^{1,2} \cdot$ Ya-Chung Tian $^{1,2}$. \\ Chih-Hsiang Chang ${ }^{1,2}$
}

Received: 27 April 2021 / Accepted: 17 September 2021 / Published online: 8 October 2021

(c) Italian Society of Nephrology 2021

\begin{abstract}
Backgrounds Neutrophil-to-lymphocyte ratio (NLR), a surrogate marker of systemic response to physiological stress, is used for prognosis prediction in many diseases. However, the usefulness of this marker for predicting acute kidney injury (AKI) progression is unclear.

Methods This retrospective study was based on the Chang Gung Research Database. Patients admitted to the intensive care unit with a diagnosis of stage 1 or 2 AKI were identified. The primary outcome was a composite of progression to stage 3 AKI, requirement of renal replacement therapy, or 14-day in-hospital mortality. The association between NLR and the primary outcome was examined using a logistic regression model and multivariable analysis. The nonlinearity and cutoff points of this relationship were determined using a restricted cubic spline model.

Results A total of 10,441 patients were enrolled. NLR level at the time of stage 1-2 AKI diagnosis was a marker of adverse outcomes. After adjustment for confounders, NLR was independently associated with the composite outcome of AKI progression, renal replacement therapy, or mortality. The restricted cubic spline model revealed a J-shaped curve, with the lowest odds ratio for an NLR between 7 and 38. Subgroup analysis revealed linear and J-shaped relationships between NLR and the primary outcome in patients admitted to the intensive care unit for medical reasons and for cardiovascular surgery, respectively.

Conclusions NLR is an independent marker of AKI progression and in-hospital mortality. Because it is readily available in daily practice, it might be used for risk stratification in the AKI population.
\end{abstract}

Chih-Hsiang Chang

franwisandsun@gmail.com

1 Department of Nephrology, Chang Gung Memorial Hospital, Linkou, Taiwan

2 Department of Nephrology, Kidney Research Center, Linkou Chang Gung Memorial Hospital, Taipei, Taiwan 


\section{Graphic abstract}

\section{Neutrophil-to-lymphocyte ratio for AKI progression or mortality prediction in critical illness, AKI population}

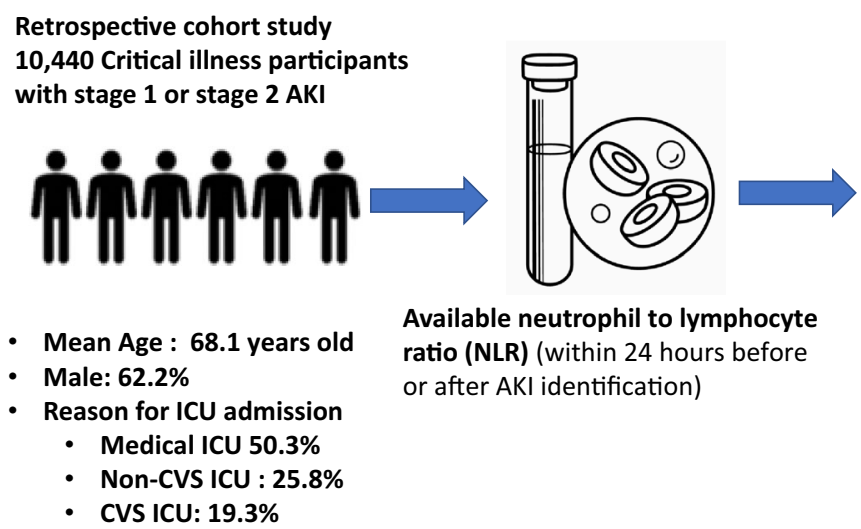

Retrospective cohort study Critical illness participants

- CVS ICU: $19.3 \%$
NLR for prediction of primary Outcome : AKI stage progression (stage 3 or dialysis requiring $\mathrm{AKI}$ ) or mortality

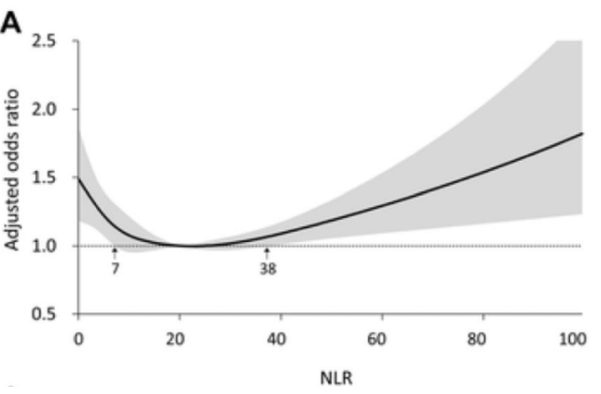

NLR $<7$ or $>38$ : higher risk of the primary composite outcome within 14 days after AKI

Keywords AKI $\cdot$ NLR $\cdot$ Outcome $\cdot$ Prediction

\author{
Abbreviations \\ AKI Acute kidney injury \\ ICU Intensive care unit \\ NLR Neutrophil-to-lymphocyte ratio \\ RRT Renal replacement therapy
}

\section{Introduction}

Acute kidney injury (AKI) is a common complication among patients admitted to the hospital. The incidence of AKI in critical illness is $20-50 \%$, and the condition has a high mortality rate [1]. The incidence of severe AKI requiring dialysis has increased in recent years [2]. Because of the high rates of morbidity and mortality associated with AKI, many studies have investigated the potential of novel biomarkers in early diagnosis or risk stratification [3-9]. However, the methods for measuring most biomarkers are expensive and might not be suitable in daily practice. The furosemide stress test, a cheap and readily available functional test, has shown promise in AKI progression prediction [10-12]. Neutrophilto-lymphocyte ratio (NLR), which can be readily measured in daily clinical practice, is calculated using the complete blood count. It is a surrogate marker of systemic response to physiological stress. NLR is associated with adverse outcomes in many diseases [13-19] and can help in early predicting AKI [20-22]. In a study of 10,859 patients admitted to an intensive care unit (ICU), Zheng et al. [23] noted that platelet-to-lymphocyte ratio, an inflammatory marker similar to NLR, exhibited a U-shaped relationship with mortality in patients with critical illness and AKI. Some studies have reported contradictory results regarding the linear relationship between NLR and outcomes [13, 14, 18, 24]. Furthermore, whether NLR could serve as a predictive marker of AKI progression remains unclear.

Therefore, we explored the ability of NLR to predict AKI progression and the association between NLR and adverse outcomes through nonlinear statistical analysis.

\section{Materials and methods}

\section{Data source}

This retrospective study was based on electronic data from the Chang Gung Research Database (CGRD). The Chang Gung Medical Foundation is the largest medical system in Taiwan and comprises seven hospitals across Taiwan. The CGRD is a multi-institutional electronic medical record system containing detailed clinical information, such as laboratory examination results, hemodynamic records, and physical examination records. The CGRD exhibits high overall and disease-specific coverage in Taiwan, and the data have been validated $[25,26]$. Disease diagnoses in the database before 2015 are based on International Classification of Diseases, Ninth Revision, Clinical Modification codes, and those after 2016 are based on International Classification of Diseases, Tenth Revision, Clinical Modification codes. This 
study was approved by the Institutional Review Board of Chang Gung Memorial Hospital (IRB No.: 202000915B0). The need for individual consent was waived because no personal identification data are included in the database.

\section{Study population}

Adult patients (age $>18$ years) who were admitted to the ICU and developed AKI in the ICU between Jan 1, 2007, and May 31, 2019, were included. When a patient had two or more ICU admissions during the study period, the first ICU admission date was chosen as the index admission. AKI was identified in accordance with the Kidney Disease: improving Global Outcomes (KDIGO) Clinical Practice Guideline for Acute Kidney Injury, that is, by comparing the baseline creatinine level with the highest creatinine level during the first 7 days of the index ICU admission [27, 28]. The baseline creatinine levels were represented by the lowest creatinine level between 7 days and 1 year before the index ICU admission (priority) or the lowest creatinine level within 6 days before the index ICU admission [29, 30]. Eligible patients were those with a diagnosis of stage 1 or 2 AKI (see Additional file 1: Supplementary Fig. 1).

The exclusion criteria were as follows: renal transplant before admission; dialysis before admission; dialysis during hospital admission but before ICU admission; stage 5 chronic kidney disease (CKD) before admission; no baseline creatinine data; AKI developed during hospital admission but before ICU admission; mortality within 2 days after ICU admission; a stage 3 AKI diagnosis or renal replacement therapy (RRT) within 8 days of ICU admission; and a diagnosis of liver cirrhosis, cancer, or autoimmune disease. Participants identified with hospital admission in the previous 1 month were excluded. NLR at the onset of stage 1 or
2 AKI during ICU admission was calculated by dividing the absolute neutrophil count by the absolute lymphocyte count obtained from a complete blood count with differential. Patients without NLR data were excluded (Fig. 1).

\section{Outcomes}

The primary outcome was a composite of AKI progression to stage 3, RRT, or in-hospital mortality within 14 days after diagnosis of stage 1 or 2 AKI. The secondary outcomes were the individual components of the primary composite outcome. Additional follow-up outcomes, including all-cause mortality, RRT, and a composite outcome of all-cause mortality or RRT during the 180-day follow-up after the onset of stage 1 or 2 AKI during ICU admission, were also investigated.

\section{Covariates}

The covariates we considered were demographics (age, sex, and body mass index), reason for ICU admission [medical admission, cardiovascular surgery (CVS), or non-cardiovascular surgery], comorbidities (hypertension, diabetes mellitus, heart failure, coronary artery disease, and CKD), and baseline creatinine level. Laboratory parameters measured on the ICU admission date included blood urea nitrogen (BUN), serum creatinine, serum albumin, C-reactive protein, white blood count (WBC) with neutrophil percentage, hemoglobin, potassium, and $\mathrm{pH}$ obtained from arterial blood gas analysis. The severity of critical illness at the onset of stage 1 or 2 AKI was assessed using sequential organ failure assessment scores (SOFA) and Acute Physiology and Chronic Health Evaluation II (APACHE-II) scores. Ventilator use on the ICU admission date and inotropic agent
Fig. 1 Flowchart of the inclusion and exclusion of patients. $A K I$ acute kidney injury,

$I C U$ intensive care unit, $N L R$ neutrophil-to-lymphocyte ratio

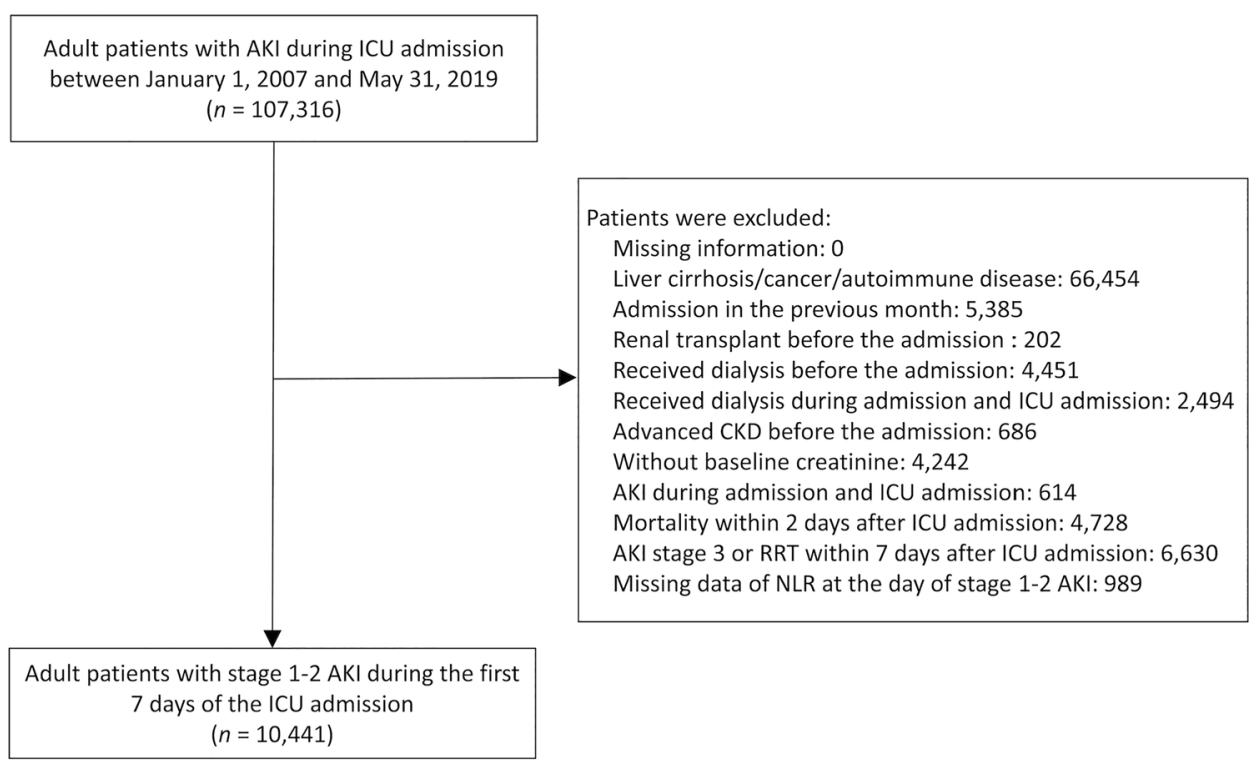


prescription at the onset of stage 1 or 2 AKI were also recorded and analyzed.

\section{Statistical analysis}

The associations between NLR and the risks of primary and secondary outcomes (e.g., progression to stage $3 \mathrm{AKI}$ ) within 14 days after the onset of stage 1 or 2 AKI were assessed using logistic regression models. NLR was divided into quintiles [quintile $1, \leq 4.7$; quintile 2, 4.8-7.9; quintile 3, 8.0-12.1; quintile 4, 12.2-20.5 (reference); and quintile $5,>20.5]$ and treated as a categorical variable. The risks of time-to-event outcomes in the NLR groups were compared using a Cox proportional hazards model. In addition to the univariate analysis (NLR as the only explanatory variable), multivariable analyses were conducted to determine whether NLR was independently associated with the outcomes of interest. Model 2 was adjusted for age and sex, and Model 3 was adjusted for hypertension, diabetes, heart failure, coronary artery disease, CKD, APACHE-II score, SOFA score, baseline creatinine, albumin, C-reactive protein, WBC, hemoglobin, potassium, BUN, pH, ventilator use, and inotropic agent use.

The continuous NLR data were employed in restricted cubic spline (RCS) regression models. The possibility of nonlinearity and the potential cutoffs for NLR were explored with the RCS model. Four knots were employed, located at the 5th, 35th, 65th, and 95th percentiles. Finally, a subgroup analysis was conducted to assess whether the linear and nonlinear effects of NLR on the primary outcome (composite of stage 3 AKI, RRT, or 14-day in-hospital mortality) was consistent across all reasons for ICU admission (including for CVS, non-cardiovascular surgery, or medical reasons).

Because of the missing values for APACHE-II score, albumin, C-reactive protein, WBC, hemoglobin, potassium, BUN, and $\mathrm{pH}$, a single expectation-maximization imputation was performed before adjustment of the covariates in the aforementioned regression models. RCS modeling was performed with R (version 4.0.2; R Foundation for Statistical Computing) and the rms package (version 5.1-3.1; Frank E. Harrell Jr.). Other statistical analyses were performed using SAS (version 9.4; SAS Institute, Cary, NC, USA). A two-sided $P$ value of $<0.05$ was considered statistically significant.

\section{Results}

\section{Patient characteristics}

A total of 107,316 patients with stage 1 or 2 AKI during ICU admission were identified. After the exclusion criteria were applied, 10,441 patients were eligible for analysis. These patients included 6491 men $(62.2 \%$; mean age, $68.1 \pm 16.6$ years). Among the patients, $5256(50.3 \%)$ were admitted for (nonsurgical) medical reasons, 2011 (19.3\%) were admitted for CVS, and 2690 (25.8\%) were admitted for non-cardiovascular surgery. The overall mean NLR was 14.9 ( \pm 17.8$)$. Patients with a higher NLR tended to be older; be men; have lower body mass indices; be less likely to have hypertension, diabetes mellitus, coronary artery disease, or CKD; have lower albumin and hemoglobin levels and higher C-reactive protein levels and WBCs at ICU admission; and be prone to requiring ventilator or inotropic agent support (Additional File 2).

\section{Association of NLR with AKI progression, RRT, and in-hospital mortality}

When NLR was considered a categorical variable, the highest quintile of NLR (NLR > 20.5) was associated with higher risks of the primary composite outcome [odds ratio [OR]: 1.36, 95\% confidence interval (CI): 1.14-1.63], AKI progression (OR: 1.38, 95\% CI: 1.03-1.84), and in-hospital mortality (OR: $1.18,95 \%$ CI: 1.01-1.36) than was the reference group (quintile $4,12.2<\mathrm{NLR}<20.5$ ). Compared with the reference, lower NLR quintiles were associated with higher risks of AKI progression and the primary composite outcome. However, no associations between NLR and the risk of requiring RRT were observed (Table 1).

When a reference NLR of 20 was used, the RCS revealed J-shaped curves for the relationships of NLR with the primary composite outcome, AKI progression, and in-hospital mortality. An NLR $<7$ or $>38$ was associated with a higher risk of the primary composite outcome than was the reference NLR (Fig. 2A). In addition, an NLR $<12$ or $>20$ was associated with a higher risk of AKI progression (Fig. 2B). Notably, only an NLR $>28$ was associated with a higher risk of in-hospital mortality (Fig. 2C). However, no J-shaped relationship between NLR and the risk of RRT was apparent, and no cutoffs were identified (Fig. 2D).

Subgroup analysis by reason for ICU admission revealed different relationships between NLR and the primary composite outcome. In the medical admission population, a generally linear relationship between NLR and the risk of primary outcome was observed (Fig. 3A). 
Table 1 Association of neutrophil-to-lymphocyte ratio quintiles at onset of stage 1 or 2 acute kidney injury during intensive care unit admission with risks of in-hospital events within 14 days

\begin{tabular}{|c|c|c|c|c|c|}
\hline \multirow[t]{2}{*}{ Outcome/NLR } & \multirow[t]{2}{*}{ Patient number } & \multirow[t]{2}{*}{ Event $(\%)$} & \multicolumn{3}{|l|}{ Odds ratio $(95 \% \mathrm{CI})$} \\
\hline & & & Model 1 & Model 2 & Model 3 \\
\hline \multicolumn{6}{|c|}{ Progress to stage $3 \mathrm{AKI}, \mathrm{RRT}$ and death } \\
\hline Quintile $1(\leq 4.7)$ & 2087 & $295(14.1)$ & $1.19(0.99-1.42)$ & $1.19(0.99-1.42)$ & $1.41(1.16-1.70)^{*}$ \\
\hline Quintile $2(4.8-7.9)$ & 2089 & $318(15.2)$ & $1.29(1.08-1.54)^{*}$ & $1.31(1.09-1.56)^{*}$ & $1.48(1.23-1.77)^{*}$ \\
\hline Quintile $3(8.0-12.1)$ & 2093 & $296(14.1)$ & $1.19(0.99-1.42)$ & $1.20(1.003-1.44)^{*}$ & $1.25(1.04-1.51)^{*}$ \\
\hline Quintile 4 (12.2-20.5) & 2082 & $254(12.2)$ & Reference & Reference & Reference \\
\hline Quintile 5 (>20.5) & 2089 & $363(17.4)$ & $1.51(1.27-1.80)^{*}$ & $1.49(1.25-1.77)^{*}$ & $1.36(1.14-1.63)^{*}$ \\
\hline \multicolumn{6}{|c|}{ Progression to AKI stage 3 (including RRT) } \\
\hline Quintile $1(\leq 4.7)$ & 2087 & $95(4.6)$ & $1.16(0.86-1.57)$ & $1.17(0.86-1.58)$ & $1.27(0.93-1.73)$ \\
\hline Quintile $2(4.8-7.9)$ & 2089 & $116(5.6)$ & $1.43(1.07-1.92)^{*}$ & $1.46(1.09-1.94)^{*}$ & $1.52(1.13-2.04)^{*}$ \\
\hline Quintile $3(8.0-12.1)$ & 2093 & $99(4.7)$ & $1.21(0.90-1.63)$ & $1.23(0.91-1.65)$ & $1.23(0.91-1.66)$ \\
\hline Quintile 4 (12.2-20.5) & 2082 & $82(3.9)$ & Reference & Reference & Reference \\
\hline Quintile 5 (> 20.5) & 2089 & $121(5.8)$ & $1.50(1.13-2.00)^{*}$ & $1.47(1.10-1.96)^{*}$ & $1.38(1.03-1.84)^{*}$ \\
\hline \multicolumn{6}{|l|}{ Progression to RRT } \\
\hline Quintile $1(\leq 4.7)$ & 2087 & $24(1.1)$ & $1.00(0.57-1.76)$ & $0.99(0.56-1.76)$ & $1.14(0.63-2.05)$ \\
\hline Quintile 2 (4.8-7.9) & 2089 & $24(1.1)$ & $1.00(0.56-1.76)$ & $1.00(0.57-1.77)$ & $1.09(0.61-1.95)$ \\
\hline Quintile $3(8.0-12.1)$ & 2093 & $19(0.9)$ & $0.79(0.43-1.44)$ & $0.79(0.43-1.46)$ & $0.81(0.44-1.50)$ \\
\hline Quintile 4 (12.2-20.5) & 2082 & $24(1.2)$ & Reference & Reference & Reference \\
\hline Quintile 5 (> 20.5) & 2089 & $35(1.7)$ & $1.46(0.87-2.47)$ & $1.44(0.85-2.43)$ & $1.35(0.79-2.31)$ \\
\hline \multicolumn{6}{|l|}{ In-hospital death } \\
\hline Quintile $1(\leq 4.7)$ & 2087 & $417(20.0)$ & $0.87(0.75-1.01)$ & $0.86(0.74-1.003)$ & $1.07(0.91-1.25)$ \\
\hline Quintile 2 (4.8-7.9) & 2089 & $434(20.8)$ & $0.91(0.79-1.05)$ & $0.93(0.80-1.08)$ & $1.07(0.91-1.25)$ \\
\hline Quintile $3(8.0-12.1)$ & 2093 & $437(20.9)$ & $0.92(0.79-1.06)$ & $0.94(0.81-1.09)$ & $0.98(0.84-1.15)$ \\
\hline Quintile 4 (12.2-20.5) & 2082 & $466(22.4)$ & Reference & Reference & Reference \\
\hline Quintile 5 (>20.5) & 2089 & $596(28.5)$ & $1.39(1.20-1.59)^{*}$ & $1.33(1.16-1.53)^{*}$ & $1.18(1.01-1.36)^{*}$ \\
\hline
\end{tabular}

Model 1 univariate model, Model 2 adjusted for age and sex, Model 3 further adjusted for hypertension, diabetes, heart failure, coronary artery disease, chronic kidney disease, Acute Physiology and Chronic Health Evaluation II score, baseline creatinine, albumin, C-reactive protein, white blood count, hemoglobin, potassium, blood urea nitrogen, $\mathrm{pH}$, ventilator use at the date of ICU admission, and inotropic agent use at the onset of stage 1 or 2 AKI during ICU admission

$N L R$ neutrophil-to-lymphocyte ratio, $A K I$ acute kidney injury, $I C U$ intensive care unit, $R R T$ renal replacement therapy, $C I$ confidence interval

$* P$ value $<0.05$

A J-shaped relationship was observed for the CVS admission population. An NLR $<7$ or $>33$ was associated with a higher risk of the primary composite outcome than was the reference (Fig. 3B), and a nonlinear relationship approximating a J-shaped curve was observed in the population admitted for non-cardiovascular surgery (Fig. 3C).

\section{Association between NLR and outcomes at 180-day follow-up}

The highest NLR quintile (NLR $>20.5$ ) was associated with higher risks of all-cause mortality [hazard ratio (HR): 1.14, 95\% CI: $1.02-1.28$ ), RRT (HR: $1.33,95 \%$ CI: $1.04-1.70]$, and of the composite of mortality or RRT (HR: 1.15, 95\% CI: 1.03-1.28; Additional file 3: Supplementary Table 2) within 180 days. With a reference NLR of 21 , the RCS model revealed J-shaped relationships between NLR and all-cause mortality and the composite of RRT and mortality (Additional file 4: Supplementary Fig. 2A and Additional file 6: Supplementary Fig. 2C). However, a nonlinear relationship was observed between NLR and dialysis for NLR $<21$, and a linear relationship was found for NLR $>21$ (Additional file 5: Supplementary Fig. 2B).

\section{Discussion}

In this large cohort study of 10,441 patients with critical illness, we examined the relationships between NLR and short- and medium-term adverse outcomes. We present the following findings: (1) NLR measured at the onset of stage 1 or stage 2 AKI predicted the primary composite outcome in 


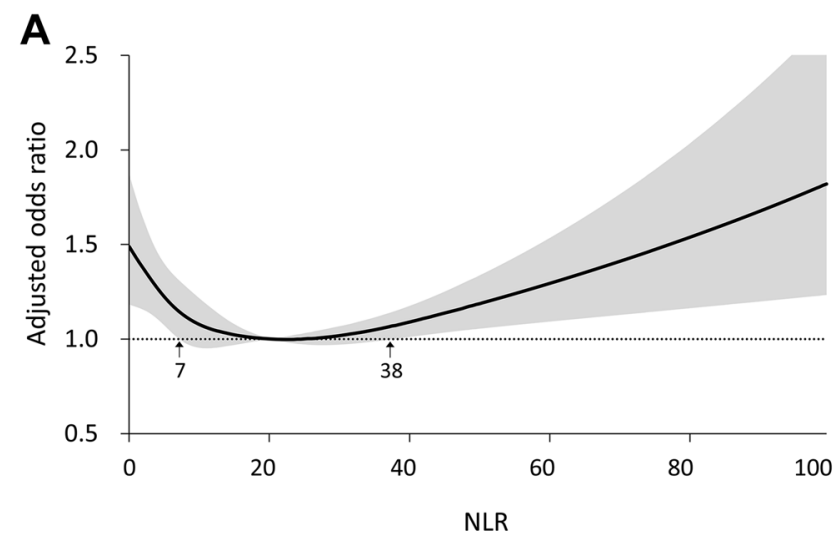

C

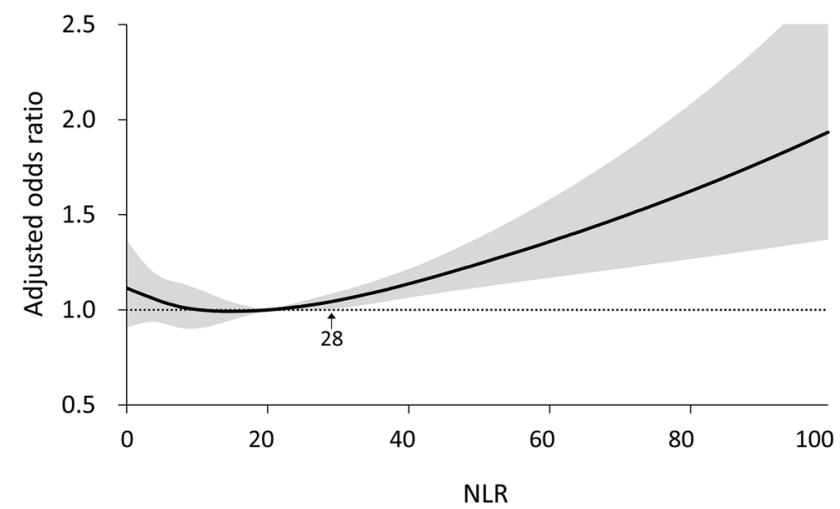

Fig. 2 Relationships between neutrophil-to-lymphocyte ratio and risks of $\mathbf{A}$ the primary composite of stage 3 acute kidney injury, renal replacement therapy, or death; $\mathbf{B}$ stage 3 acute kidney injury including renal replacement therapy; $\mathbf{C}$ in-hospital mortality; and $\mathbf{D}$ renal

the short term, which included in-hospital mortality and AKI progression. (2) NLR and the composite outcome exhibited a J-shaped relationship in the short term, with the lowest OR observed for an NLR between 7 and 38. (3) Subgroup analysis revealed a $\mathrm{J}$-shaped association between NLR and short-term adverse outcomes among patients admitted for surgery, especially among those admitted for CVS, with the lowest OR observed for an NLR between 7 and 33. (4) NLR at the onset of stage 1 or stage 2 AKI also predicted the secondary composite of RRT or mortality in the medium term (180 days), with a J-shaped relationship and the lowest OR observed for an NLR between 5 and 21 .

In response to physiological stress including infection or inflammation, endogenous cortical catecholamines increase, thereby raising the neutrophil count and resulting in lymphopenia [31, 32]. Therefore, physiological stress may increase NLR, which could thus serve as an indicator of systemic stress response. NLR rapidly increases within $6 \mathrm{~h}$ after physiologic stress [33] and indicates a response earlier than total WBC and left shift on WBC differential analysis [34]. NLR has been used to distinguish mild and severe disease and as a prognostic marker. For example, Hajibandeh et al.

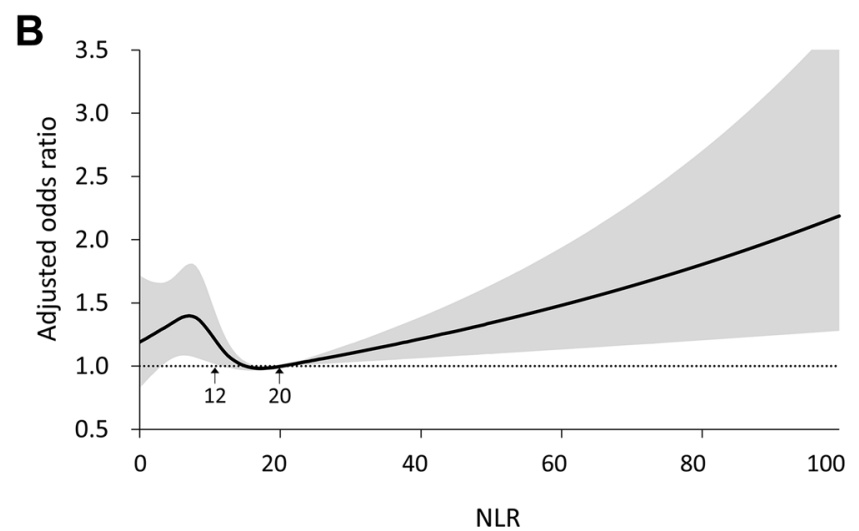

D

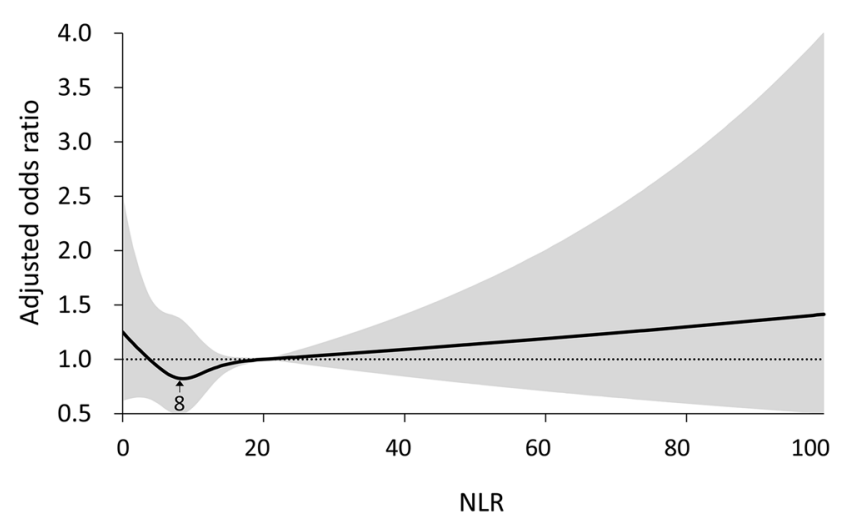

replacement therapy within 14 days after the onset of stage 1 or 2 acute kidney injury during intensive care unit admission. NLR neutrophil-to-lymphocyte ratio, $A K I$ acute kidney injury, $R R T$ renal replacement therapy, $I C U$ intensive care unit

determined that NLR could be used as a diagnostic tool for both early diagnosis and severity detection of appendicitis in those presenting with right lower quadrant pain [35]. According to Yazar, using NLR and platelet-to-lymphocyte ratio in addition to routine diagnostic tools could increase the accuracy of appendicitis diagnosis in pregnant women [36].

We observed a J-shaped relationship between in-hospital mortality and NLR during early-stage AKI. NLR could also be used as a prognostic marker in various diseases (including pulmonary embolism, pancreatitis, sepsis, etc.), and some studies have demonstrated a linear relationship [13-17, 24]. Other studies have demonstrated J-shaped relationships of NLR and cortisol levels with patient survival $[18,37]$. Salciccioli et al. reported a stepwise increase in 28-day mortality with an increase in NLR in a critically ill population, but this linear relationship was not observed in patients with sepsis [16]. According to Hwang et al., in people with septic shock, persistently low NLR is associated with the worst outcome [18]. A similar hemogram parameter, platelet-tolymphocyte ratio, was reported to have a J-shaped relationship with mortality in patients on dialysis [38]. These results 

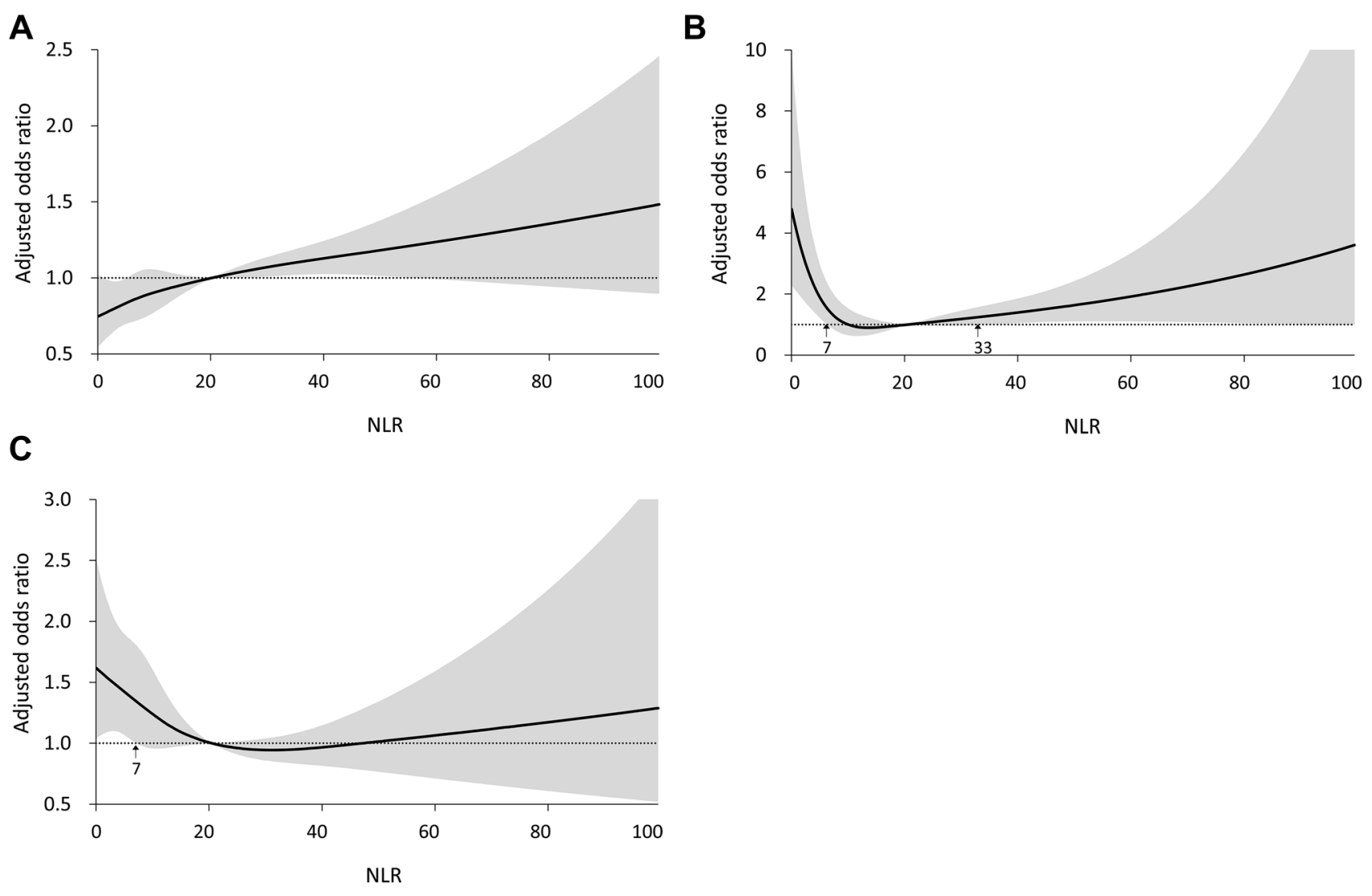

Fig. 3 Relationships between neutrophil-to-lymphocyte ratio and risks of primary composite outcome within 14 days after the onset of stage 1 or 2 acute kidney injury during intensive care unit admis-

sion for $\mathbf{A}$ medical reasons, $\mathbf{B}$ cardiovascular surgery, and $\mathbf{C}$ major non-cardiovascular surgery. $N L R$ neutrophil-to-lymphocyte ratio, $A K I$ acute kidney injury, ICU intensive care unit

imply that a response of normal or low NLR to physiological stress might be maladaptive and thus result in an unfavorable outcome. The present study revealed that in patients with early-stage AKI and an NLR $<7$ or $>38$ is associated with in-hospital adverse events, including AKI progression and mortality, in the short term.

In subgroup analysis, we revealed a linear relationship between composite outcome of AKI mortality or mortality and NLR in the medical ICU population. Similar findings have also been reported in a pulmonary embolism population (NLR cut-off value for mortality ranged from 5.4 to 9.2) [13], in a pancreatitis population (NLR cut-off value for severe pancreatitis: 9.6 with sensitivity: $81.7 \%$, specificity: $55.3 \%$ and AUROC: 0.72 at day 0) [14], and in a CKD population (HR for all-cause mortality: 1.45, HR for cardiovascular event: 1.52) [39]. In septic patients, the relationship between the NLR and mortality is more complex. Hwang et al. reported that the relationship between NLR and mortality is linear in a subgroup with increasing NLR, and that it is a $\mathbf{J}$ curve relationship in a subgroup with stable NLR [18]. However, in mixed medical and surgical populations, we revealed a non-linear relationship between NLR and

composite outcome. If we apply a traditional linear relationship to our overall cohort, the AUROC of NLR for outcome prediction is poor (AUROC: 0.51 for composite outcome of AKI progression and death; data not shown).

The J-shaped association between AKI progression and NLR we observed has not been demonstrated previously. Several trials have reported that NLR could be used as a marker of AKI in patients after CVS [21], in individuals presenting at emergency departments [40], in patients receiving emergency coronary angiography [22], and in patients with sepsis [41]; however, these trials have used small patient samples. NLR has also been demonstrated as a marker of CKD progression [42], a marker of CV mortality [43], and a marker of arteriovenous fistula stenosis in end-stage renal disease [44].

In this study, we investigated the association between NLR and short-and medium-term adverse outcomes. To our knowledge, this is the first study to demonstrate NLR to be a marker of AKI progression. The strengths of our study include its multi-institutional data and large scale. Moreover, we examined the associations of NLR and adverse outcomes by using the RCS function, which 
has rarely been used in related studies. Furthermore, we enrolled a diverse population and conducted subgroup analysis. However, the present study has some limitations that should be considered when the results are interpreted. First, this was a retrospective cohort study, and despite adjustment for potential confounding factors derived from previous studies $[45,46]$, residual confounding might still exist. Second, some diseases or clinical conditions that could result in lymphopenia or atypical lymphocytosis [47-49] were not identified in our cohort; thus, the diagnostic value of NLR might be misrepresented. Third, we did not track the relationships between NLR trajectory and adverse outcomes. Fourth, we simply divided the blood neutrophil count by the lymphocyte count, and atypical lymphocytes and lymphocyte subsets were not considered in our analysis. Further prospective study is needed for validating the prognostic role of NLR in critical illness patients with AKI. Moreover, the diagnostic benefits of including NLR in conventional critical illness scoring systems or AKI severity prediction should be evaluated.

\section{Conclusion}

The present study suggests that NLR can serve as an independent marker of AKI progression and in-hospital adverse events, including mortality. NLR shared a $\mathrm{J}$-shaped relationship with both short- and medium-term outcomes. NLR is a simple, cost-effective, and readily available marker that could be used for early risk stratification in critical illness patients with acute kidney injury.

Supplementary Information The online version contains supplementary material available at https://doi.org/10.1007/s40620-021-01162-3.

Acknowledgements The authors thank Mr. Alfred Hsing-Fen Lin for assistance in the statistical analysis. This study is based in part on data from the Chang Gung Research Database provided by Chang Gung Memorial Hospital. The interpretation and conclusions contained herein do not represent the position of Chang Gung Memorial Hospital.

Author's contributions JJC, CHC and GK methodology; CCL, GK and CLY, formal analysis; JJC and THL data extraction; JJC and THL, writing - original draft preparation; YCT, CHC and PHC writing - review and editing; $\mathrm{CHC}$ project administration. All authors read and approved the final manuscript.

Funding This study was supported by grants from Chang Gung Memorial Hospital, Taiwan, (CMRPG5K0141), and C-H Chang was supported by the Ministry of Science and Technology (109-2314-B-182A-124).

Availability of data and materials Not applicable.

Code availability Not applicable.

\section{Declarations}

Conflict of interest The authors declare that they have no competing interests.

Ethical approval Approved by the Institutional Review Board of Chang Gung Memorial Hospital (IRB No.: 202000915B0).

Consent to participate The need for individual consent was waived.

Consent for publication Not applicable.

\section{References}

1. Case J, Khan S, Khalid R, Khan A (2013) Epidemiology of acute kidney injury in the intensive care unit. Crit Care Res Pract 2013:479730. https://doi.org/10.1155/2013/479730

2. Harding JL, Li Y, Burrows NR, Bullard KM, Pavkov ME (2020) US trends in hospitalizations for dialysis-requiring acute kidney injury in people with versus without diabetes. Am J Kidney Dis 75(6):897-907. https://doi.org/10.1053/j.ajkd.2019.09.012

3. Klein SJ, Brandtner AK, Lehner GF et al (2018) Biomarkers for prediction of renal replacement therapy in acute kidney injury: a systematic review and meta-analysis. Intensive Care Med 44(3):323-336. https://doi.org/10.1007/s00134-018-5126-8

4. Zhang A, Cai Y, Wang PF et al (2016) Diagnosis and prognosis of neutrophil gelatinase-associated lipocalin for acute kidney injury with sepsis: a systematic review and meta-analysis. Crit Care 20:41. https://doi.org/10.1186/s13054-016-1212-x

5. Chang $\mathrm{CH}$, Yang $\mathrm{CH}$, Yang HY et al (2015) Urinary biomarkers improve the diagnosis of intrinsic acute kidney injury in coronary care units. Medicine (Baltimore) 94(40):e1703. https://doi.org/10. 1097/MD.0000000000001703

6. Lee CC, Chang CH, Cheng YL et al (2019) Diagnostic performance of cyclophilin a in cardiac surgery-associated acute kidney injury. J Clin Med 9(1):108. https://doi.org/10.3390/jcm9010108

7. Jia HM, Huang LF, Zheng Y, Li WX (2017) Diagnostic value of urinary tissue inhibitor of metalloproteinase-2 and insulinlike growth factor binding protein 7 for acute kidney injury: a meta-analysis. Crit Care 21(1):77. https://doi.org/10.1186/ s13054-017-1660-y

8. Albert C, Zapf A, Haase M et al (2020) Neutrophil gelatinaseassociated lipocalin measured on clinical laboratory platforms for the prediction of acute kidney injury and the associated need for dialysis therapy: a systematic review and meta-analysis. Am J Kidney Dis 76(6):826-841 e821. https://doi.org/10.1053/j.ajkd. 2020.05.015

9. Wu VC, Shiao CC, Chi NH et al (2018) Outcome prediction of acute kidney injury biomarkers at initiation of dialysis in critical units. J Clin Med 7(8):202. https://doi.org/10.3390/jcm7080202

10. Chawla LS, Davison DL, Brasha-Mitchell E et al (2013) Development and standardization of a furosemide stress test to predict the severity of acute kidney injury. Crit Care 17(5):R207. https://doi. org/10.1186/cc13015

11. Chen JJ, Chang CH, Huang YT, Kuo G (2020) Furosemide stress test as a predictive marker of acute kidney injury progression or renal replacement therapy: a systemic review and meta-analysis. Crit Care 24(1):202. https://doi.org/10.1186/s13054-020-02912-8

12. Chawla LS, Ronco C (2016) Renal stress testing in the assessment of kidney disease. Kidney Int Rep 1(1):57-63. https://doi.org/10. 1016/j.ekir.2016.04.005

13. Wang Q, Ma J, Jiang Z, Ming L (2018) Prognostic value of neutrophil-to-lymphocyte ratio and platelet-to-lymphocyte ratio in acute 
pulmonary embolism: a systematic review and meta-analysis. Int Angiol 37(1):4-11. https://doi.org/10.23736/S0392-9590.17. 03848-2

14. Han C, Zeng J, Lin R et al (2017) The utility of neutrophil to lymphocyte ratio and fluid sequestration as an early predictor of severe acute pancreatitis. Sci Rep 7(1):10704. https://doi.org/10. 1038/s41598-017-10516-6

15. Jeon TJ, Park JY (2017) Clinical significance of the neutrophillymphocyte ratio as an early predictive marker for adverse outcomes in patients with acute pancreatitis. World J Gastroenterol 23(21):3883-3889. https://doi.org/10.3748/wjg.v23.i21.3883

16. Salciccioli JD, Marshall DC, Pimentel MA et al (2015) The association between the neutrophil-to-lymphocyte ratio and mortality in critical illness: an observational cohort study. Crit Care 19:13. https://doi.org/10.1186/s13054-014-0731-6

17. Park HJ, Woo SH, Kim DH et al (2021) Efficacy of blood urea nitrogen and the neutrophil-to-lymphocyte ratio as predictors of mortality among elderly patients with genitourinary tract infections: a retrospective multicentre study. J Infect Chemother 27(2):312-318. https://doi.org/10.1016/j.jiac.2020.11.007

18. Hwang SY, Shin TG, Jo IJ et al (2017) Neutrophil-to-lymphocyte ratio as a prognostic marker in critically-ill septic patients. Am J Emerg Med 35(2):234-239. https://doi.org/10.1016/j.ajem.2016. 10.055

19. Yodying H, Matsuda A, Miyashita M et al (2016) Prognostic significance of neutrophil-to-lymphocyte ratio and platelet-tolymphocyte ratio in oncologic outcomes of esophageal cancer: a systematic review and meta-analysis. Ann Surg Oncol 23(2):646654. https://doi.org/10.1245/s10434-015-4869-5

20. Valga F, Monzon T, Henriquez F, Anton-Perez G (2019) Neutrophil-to-lymphocyte and platelet-to-lymphocyte ratios as biological markers of interest in kidney disease. Nefrologia (Engl Ed) 39(3):243-249. https://doi.org/10.1016/j.nefro.2018.11.005

21. Kim WH, Park JY, Ok SH, Shin IW, Sohn JT (2015) Association between the neutrophil/lymphocyte ratio and acute kidney injury after cardiovascular surgery: a retrospective observational study. Medicine (Baltimore) 94(43):e1867. https://doi.org/10.1097/MD. 0000000000001867

22. Yuan Y, Qiu H, Hu X et al (2017) Predictive value of inflammatory factors on contrast-induced acute kidney injury in patients who underwent an emergency percutaneous coronary intervention. Clin Cardiol 40(9):719-725. https://doi.org/10.1002/clc. 22722

23. Zheng CF, Liu WY, Zeng FF et al (2017) Prognostic value of platelet-to-lymphocyte ratios among critically ill patients with acute kidney injury. Crit Care 21(1):238. https://doi.org/10.1186/ s13054-017-1821-z

24. Bajaj A, Saleeb M, Rathor P, Sehgal V, Kabak B, Hosur S (2015) Prognostic value of troponins in acute nonmassive pulmonary embolism: a meta-analysis. Heart Lung 44(4):327-334. https:// doi.org/10.1016/j.hrtlng.2015.03.007

25. Tsai MS, Lin MH, Lee CP et al (2017) Chang Gung Research Database: a multi-institutional database consisting of original medical records. Biomed J 40(5):263-269. https://doi.org/10. 1016/j.bj.2017.08.002

26. Shao SC, Chan YY, Kao Yang YH et al (2019) The Chang Gung Research Database-a multi-institutional electronic medical records database for real-world epidemiological studies in Taiwan. Pharmacoepidemiol Drug Saf 28(5):593-600. https://doi.org/10.1002/ pds. 4713

27. (2012) Section 2: AKI definition. Kidney Int Suppl (2011). 2(1): 19-36. https://doi.org/10.1038/kisup.2011.32

28. Margolis G, Gal-Oz A, Letourneau-Shesaf S, Khoury S, Keren G, Shacham Y (2018) Acute kidney injury based on the KDIGO criteria among ST elevation myocardial infarction patients treated by primary percutaneous intervention. J Nephrol 31(3):423-428. https://doi.org/10.1007/s40620-017-0461-3

29. Siew ED, Ikizler TA, Matheny ME et al (2012) Estimating baseline kidney function in hospitalized patients with impaired kidney function. Clin J Am Soc Nephrol 7(5):712-719. https://doi.org/ 10.2215/CJN.10821011

30. De Rosa S, Samoni S, Ronco C (2016) Creatinine-based definitions: from baseline creatinine to serum creatinine adjustment in intensive care. Crit Care 20:69. https://doi.org/10.1186/ s13054-016-1218-4

31. Onsrud M, Thorsby E (1981) Influence of in vivo hydrocortisone on some human blood lymphocyte subpopulations. I. Effect on natural killer cell activity. Scand J Immunol 13(6):573-579. https://doi.org/10.1111/j.1365-3083.1981.tb00171.x

32. Benschop RJ, Rodriguez-Feuerhahn M, Schedlowski M (1996) Catecholamine-induced leukocytosis: early observations, current research, and future directions. Brain Behav Immun 10(2):77-91. https://doi.org/10.1006/brbi.1996.0009

33. Zahorec R (2001) Ratio of neutrophil to lymphocyte counts-rapid and simple parameter of systemic inflammation and stress in critically ill. Bratisl Lek Listy 102(1):5-14

34. Honda T, Uehara T, Matsumoto G, Arai S, Sugano M (2016) Neutrophil left shift and white blood cell count as markers of bacterial infection. Clin Chim Acta 457:46-53. https://doi.org/ 10.1016/j.cca.2016.03.017

35. Hajibandeh S, Hajibandeh S, Hobbs N, Mansour M (2020) Neutrophil-to-lymphocyte ratio predicts acute appendicitis and distinguishes between complicated and uncomplicated appendicitis: a systematic review and meta-analysis. Am J Surg 219(1):154-163. https://doi.org/10.1016/j.amjsurg.2019.04.018

36. Yazar FM, Bakacak M, Emre A et al (2015) Predictive role of neutrophil-to-lymphocyte and platelet-to-lymphocyte ratios for diagnosis of acute appendicitis during pregnancy. Kaohsiung $\mathbf{J}$ Med Sci 31(11):591-596. https://doi.org/10.1016/j.kjms.2015.10. 005

37. Sam S, Corbridge TC, Mokhlesi B, Comellas AP, Molitch ME (2004) Cortisol levels and mortality in severe sepsis. Clin Endocrinol (Oxf) 60(1):29-35. https://doi.org/10.1111/j.1365-2265. 2004.01923.x

38. Catabay C, Obi Y, Streja E et al (2017) Lymphocyte cell ratios and mortality among incident hemodialysis patients. Am J Nephrol 46(5):408-416. https://doi.org/10.1159/000484177

39. Zhao WM, Tao SM, Liu GL (2020) Neutrophil-to-lymphocyte ratio in relation to the risk of all-cause mortality and cardiovascular events in patients with chronic kidney disease: a systematic review and meta-analysis. Ren Fail 42(1):1059-1066. https://doi. org/10.1080/0886022X.2020.1832521

40. Abu Alfeilat M, Slotki I, Shavit L (2018) Single emergency room measurement of neutrophil/lymphocyte ratio for early detection of acute kidney injury (AKI). Intern Emerg Med 13(5):717-725. https://doi.org/10.1007/s11739-017-1715-8

41. Bu X, Zhang L, Chen P, Wu X (2019) Relation of neutrophil-tolymphocyte ratio to acute kidney injury in patients with sepsis and septic shock: a retrospective study. Int Immunopharmacol 70:372-377. https://doi.org/10.1016/j.intimp.2019.02.043

42. Yuan Q, Wang J, Peng Z et al (2019) Neutrophil-to-lymphocyte ratio and incident end-stage renal disease in Chinese patients with chronic kidney disease: results from the Chinese Cohort Study of Chronic Kidney Disease (C-STRIDE). J Transl Med 17(1):86. https://doi.org/10.1186/s12967-019-1808-4

43. Lu X, Wang S, Zhang G, Xiong R, Li H (2018) High neutrophilto-lymphocyte ratio is a significant predictor of cardiovascular and all-cause mortality in patients undergoing peritoneal dialysis. Kidney Blood Press Res 43(2):490-499. https://doi.org/10.1159/ 000488696 
44. Yilmaz H, Bozkurt A, Cakmak M et al (2014) Relationship between late arteriovenous fistula (AVF) stenosis and neutrophillymphocyte ratio (NLR) in chronic hemodialysis patients. Ren Fail 36(9):1390-1394. https://doi.org/10.3109/0886022X.2014. 945183

45. Karakonstantis S, Kalemaki D, Tzagkarakis E, Lydakis C (2018) Pitfalls in studies of eosinopenia and neutrophil-to-lymphocyte count ratio. Infect Dis (Lond). 50(3):163-174. https://doi.org/10. 1080/23744235.2017.1388537

46. Matos LC, Batista P, Monteiro N et al (2013) Lymphocyte subsets in alcoholic liver disease. World J Hepatol 5(2):46-55. https://doi. org/10.4254/wjh.v5.i2.46

47. Cheng Y, Zhao H, Song P, Zhang Z, Chen J, Zhou YH (2019) Dynamic changes of lymphocyte counts in adult patients with severe pandemic H1N1 influenza A. J Infect Public Health 12(6):878-883. https://doi.org/10.1016/j.jiph.2019.05.017

48. Descamps V (2012) Drug reaction with eosinophilia and systemic symptoms (DRESS). Rev Prat 62(10):1347-1352

49. Cunha BA, Pherez F, Walls N (2009) Severe cytomegalovirus (CMV) community-acquired pneumonia (CAP) in a nonimmunocompromised host. Heart Lung 38(3):243-248. https://doi.org/10. 1016/j.hrtlng.2008.05.008

Publisher's Note Springer Nature remains neutral with regard to jurisdictional claims in published maps and institutional affiliations. 